\title{
Propiverine Hydrochloride as a Treatment for Fecal Incontinence
}

\author{
Yasue Irei ${ }^{1}$, Shota Takano ${ }^{2}$, Kazutaka Yamada ${ }^{1}$ \\ ${ }^{1}$ Department of Surgery, Coloproctology Center Takano Hospital, Kumamoto; ${ }^{2}$ Department of Functional Anorectal Disorders, \\ Coloproctology Center Takano Hospital, Kumamoto, Japan
}

Purpose: Propiverine hydrochloride (PH) is widely used for the treatment of urinary incontinence (UI) due to bladder overactivity. Moreover, the comorbidity of UI with fecal incontinence (FI) is known to be due to the relationship of both to nervous system disorders and dysfunction or weakening of the pelvic floor muscles. The aim of this single-arm prospective study was to evaluate the therapeutic value of PH for FI.

Methods: Patients $(\mathrm{n}=24)$ who were diagnosed as having both FI and UI from April 2015 to November 2016 were included in the study and administered a dosage of 10-20 mg PH every day for 1 month. The primary endpoint was to create a reduction in the frequency of FI per week. An evaluation criterion of $\geq 50 \%$ reduction in frequency was determined as effective. The percentage of the patients who achieved the $\geq 50 \%$ endpoint (responders) was also calculated.

Results: The frequency of FI per week was $6.0 \pm 8.2(0.25-30)$ at baseline and reduced to $1.6 \pm 2.1(0-7)$ at the posttherapeutic state $(\mathrm{P}=0.005)$. A reduction of $\geq 50 \%$ was seen in 14 of the patients $(58.3 \%)$.

Conclusion: PH reduced the frequency of FI in patients with both FI and UI. This study introduces a possible therapeutic option for the pharmacological treatment of FI.

Keywords: Fecal incontinence; Urinary incontinence; Propiverine hydrochloride; Pharmacological treatment

\section{INTRODUCTION}

Fecal incontinence (FI) is a medical and social problem that is reported in approximately $2.2 \%$ to $25 \%$ of the adult population [1]. Conservative treatments (i.e., dietary and pharmacological treatment) are recommended as a first-line therapy for FI [2]. Pharmacological treatments are expected to slow colonic transit, decrease intestinal fluid secretion, increase absorption, and reduce anal sphincter relaxation. Alpha-1 adrenergic agonists can reduce both bowel contraction and sphincter relaxation; however, the

Received: July 24, 2019 - Accepted: September 30, 2019

Correspondence to: Yasue Irei, M.D.

Department of Surgery, Coloproctology Center Takano Hospital, 3-2-55 0e, Chuo-ku, Kumamoto 862-0971, Japan

Tel: +81-96-320-6500, Fax: +81-96-320-6555

E-mail:ys2410uma@gmail.com

ORICD: https://orcid.org/0000-0002-8659-4211

(C) 2020 The Korean Society of Coloproctology

This is an open-access article distributed under the terms of the Creative Commons Attribution NonCommercial License (https://creativecommons.org/licenses/by-nc/4.0) which permits unrestricted non-

commercial use, distribution, and reproduction in any medium, provided the original work is properly cited. number and variety of government authorized medical treatments are limited in Japan.

Propiverine hydrochloride (PH; Fig. 1) is widely used for urinary incontinence (UI) and an overactive bladder. PH and its metabolites decrease the frequency of urination and reduce urinary bladder contraction activity by directly inhibiting the effect of calcium influx in smooth muscle cells [3], and has an anticholinergic effect that induces the weakening of contractions [4]. The comorbidity of UI with FI is known due to the relationship of both to nervous system disorders and dysfunction or weakening of the pelvic floor muscles. Therefore, the aim of this study was to evaluate the therapeutic value of $\mathrm{PH}$ for FI.

\section{METHODS}

A single-arm prospective study was conducted in the Department of Functional Anorectal Disorders at the Coloproctology Center of Takano hospital. Data were prospectively collected in patients with FI and UI as comorbidity from April 2015 to November 2016. Written informed consent based on the protocol outlined in 
the Helsinki Declaration and approved by the institution review board of the Coloproctology Center Takano Hospital (14-07) was obtained from all of the subjects before they were enrolled in the study.

\section{Patients}

Consecutive cases $(n=28)$ with soiling or urgency were diagnosed as having both FI and UI during the above-mentioned study period. Inclusion criteria for this study included patients with FI of solid stool or liquid stool and patients also having urge and/or stress UI. Exclusion criteria included patients who only had gas incontinence, patients with rectal prolapse, active anal fistula, perianal abscess, and/or patients who had undergone rectal surgery or anal sphincteroplasty within a 24-month period. Moreover, 4 patients did not come to the hospital for posttreatment follow-up and they were therefore excluded from the study. Patients with a history of treatment for FI before this study were included in this study; therefore, a total of 24 patients were enrolled.

\section{Clinical assessment}

Clinical evaluation was conducted at pretreatment and at posttreatment in the first or second follow-up visits, which were set for 3 weeks after the administration of $\mathrm{PH}$. The primary endpoint was to reach a reduction in the frequency of FI (liquid or solid stool) per week, with a $\geq 50 \%$ reduction in frequency as determined effective. The percentage of patients who achieved a reduction of $\geq 50 \%$ in frequency (responders) was calculated. The secondary endpoints were to evaluate and compare variations in the

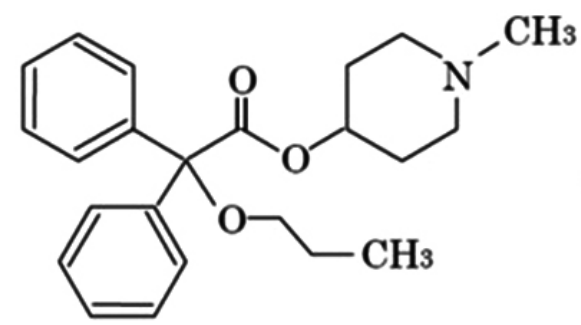

$\cdot \mathrm{HCl}$

Fig. 1. Chemical structure of propiverine hydrochloride (1-methyl4-piperidyldiphenylpropoxyacetate hydrochloride).
Fecal Incontinence Severity Index (FISI) [5], Cleveland Clinic Florida Fecal Incontinence Score (Wexner Score) [6], and the Fecal Incontinence Quality of Life Scale (JFIQL) [7] between pretreatment and posttreatment status.

A comparison of the clinical profiles between the responders and nonresponders is shown in Tables 1 and 2. The variation between pretreatment and posttreatment status for subjective UI symptoms was evaluated. The frequency of UI per week was measured, and UI symptoms were measured using the Overactive Bladder Symptom Score (OABSS) and International Consultation on Incontinence Questionnaire-Short Form (ICIQ-SF).

\section{Study design}

A registration form was used to evaluate the pre- and posttreatment status. Patients were interviewed by a trained physician to obtain information on comorbidity, operative history, past history,

Table 2. Relationship between each scale and responder/nonresponder patients

\begin{tabular}{lcccc}
\hline Variable & All patients (n) Responder (n) Nonresponder (n) & P-value \\
\hline Lifestyle & 9 & 6 & 3 & 0.392 \\
Increase & 0 & 0 & 0 & \\
No change & 3 & 2 & 1 & \\
Decrease & & & & 0.214 \\
Coping/behavior & 7 & 5 & 2 & \\
Increase & 2 & 2 & 0 & \\
No change & 3 & 1 & 2 & \\
Decrease & & & & 1.000 \\
Depression/ & 9 & 6 & 3 & \\
self-perception & & 0 & 0 & \\
Increase & 0 & 2 & 1 & \\
No change & 3 & & & \\
Decrease & 7 & 4 & 3 & \\
Embarrassment & & 0 & 1 & \\
Increase & 4 & 3 & 1 & \\
No change & 1 & & & \\
Decrease & 4 & & & \\
\hline & & & & \\
\hline
\end{tabular}

Table 1. Variation in the fecal incontinence quality of life scale between baseline and posttreatment status

\begin{tabular}{llll}
\hline Variable & \multicolumn{1}{c}{ Baseline } & Posttreatment & P-value \\
\hline Lifestyle $(n=12)$ & $2.7 \pm 0.6(1.3-3.7)$ & $2.9 \pm 0.9(1-4)$ & 0.092 \\
Coping/behavior $(n=12)$ & $2.5 \pm 0.7(1.4-4)$ & $2.7 \pm 0.8(1.1-4)$ & 0.011 \\
Depression/self-perception $(n=12)$ & $2.8 \pm 0.8(1.3-3.9)$ & $3.1 \pm 0.9(1.7-4.5)$ & 0.015 \\
Embarrassment $(n=12)$ & $2.1 \pm 0.7(1-3.5)$ & $2.6 \pm 0.8(1.3-4)$ & 0.039 \\
General score $(n=12)$ & $2.5 \pm 0.1(1.5-3.4)$ & $2.8 \pm 0.7(1.5-3.8)$ & 0.004 \\
\hline
\end{tabular}

Values are presented as mean \pm standard deviation (range). 
frequency of vaginal delivery, history of pretreatment for FI, status of UI, frequency of defecation, and the Bristol stool scale score. Information on each symptom and frequency of FI and the Quality of life (QoL) score related to FI (FISI, Wexner, JFQL) was obtained from the questionnaire and then analyzed.

When the responders provided more than one score on the Bristol stool scale, the highest score was adopted for the data. Manometry was performed at pretreatment. Maximal anal resting pressure (MRP) and maximal anal squeeze pressures (MSP) were recorded. Ultrasound examination of the anus was also performed at pretreatment and the condition of the sphincter muscles was also evaluated.

The patients were treated with a $\mathrm{PH}$ dose of 10 or $20 \mathrm{mg}$ per day. Treatment was discontinued if the patients experienced adverse events. No other pharmacological or surgical therapy was given at the start of the study period.

\section{Statistical analysis}

The nonparametric Wilcoxon test was used to evaluate the baseline and posttreatment variables. The results are either expressed individually or as the mean \pm standard deviation. Differences were considered significant when the P-value of a type I error was $\leq 0.05$. The clinical profiles were compared using the chi-square test.

\section{RESULTS}

\section{Patient profile}

Twenty-four patients met the inclusion criteria $(7$ males, $17 \mathrm{fe}-$ males). The mean age was $72.6 \pm 11.8$ (39-89) years of age. Ten patients $(41.6 \%)$ had a history of recto-anal or pelvic organ surgery (hemorrhoid surgery, ligation, and excision, 6; sphincteroplasty, 1; rectal prolapse surgery, 1; uterine prolapse surgery, 1; and total hysterectomy, 1). Fifteen of the female patients (88.2\%) experienced more than one vaginal delivery and one of them experienced perineal laceration. Ten patients (41.6\%) had a history of patient illness or comorbidities including diabetes mellitus, 3 patients; dementia, 2; myotonic dystrophy, 1; lumber spinal canal stenosis, 2; history of lumber compression fracture, 1; and a history of spine caries, 1 . Seventeen patients $(70.8 \%)$ had a history of treatments for FI before this study including biofeedback, 3 patients; Posterior Tibial Nerve Stimulation, 3; medical treatment polycarbophil calcium, 9; trimebutine maleate, 3; loperamide hydrochloride, 2; and mepenzolate bromide 1 . For complete details see Table 3.

\section{Study administration}

Twenty-one patients were administered a 20-mg dose of $\mathrm{PH}$ per day. The treatment started with a dose of $10 \mathrm{mg}$ per day for $3 \mathrm{pa}-$ tients that was then increased to $20 \mathrm{mg}$ per day for 2 of the patients 3 weeks later. Treatment was discontinued in 1 patient who was receiving a dosage of $10 \mathrm{mg}$ per day for 25 days due to dizziness. The observation period was $28 \pm 9.4$ days (14-63 days).
Table 3. Patient clinical characteristics $(\mathrm{n}=24)$

\begin{tabular}{|c|c|}
\hline Characteristic & Value \\
\hline \multicolumn{2}{|l|}{ Sex, n } \\
\hline Male & 7 \\
\hline Female (vaginal delivery experienced $>$ once) & $17(15)$ \\
\hline Age $(y r)$, mean \pm SD & $72.6 \pm 11.8$ \\
\hline \multicolumn{2}{|l|}{ History of anal and pelvic organ surgery } \\
\hline Ligation and excision & 6 \\
\hline Sphincteroplasty & 1 \\
\hline Rectal prolapse surgery & 1 \\
\hline Uterine prolapse surgery & 1 \\
\hline Total hysterectomy & 1 \\
\hline \multicolumn{2}{|l|}{ History of patient illness or comorbidity } \\
\hline Diabetes mellitus & 3 \\
\hline Dementia & 2 \\
\hline Lumber spinal canal stenosis & 2 \\
\hline Spine caries & 1 \\
\hline Myotonic dystrophy & 1 \\
\hline Lumber compression fracture & 1 \\
\hline \multicolumn{2}{|l|}{ History of treatment for Fl before this study } \\
\hline None & 7 \\
\hline Loperamide hydrochloride & 2 \\
\hline Polycarbophil calcium & 9 \\
\hline Trimebutine maleate & 3 \\
\hline Mepenzolate bromide & 1 \\
\hline Biofeedback & 3 \\
\hline PTNS & 3 \\
\hline
\end{tabular}

SD, standard deviation; Fl, fecal incontinence; PTNS, posterior tibial nerve stimulation.

Table 4. Variation in the frequency of urinary incontinence per week and symptom of urinary incontinence (OABSS, ICIQ-SF) scores between baseline and posttreatment status

\begin{tabular}{lccc}
\hline Variable & Baseline & Posttreatment & P-value \\
\hline $\begin{array}{l}\text { Frequency of Ul per } \\
\text { week }(n=13)\end{array}$ & $13.2 \pm 12.4(0.5-42)$ & $4.6 \pm 8.2(0-21)$ & 0.001 \\
OABSS $(n=11)$ & $7.5 \pm 3.6(1-13)$ & $6.6 \pm 4.1(0-12)$ & 0.228 \\
ICIQ-SF $(n=11)$ & $10.5 \pm 5.1(1-18)$ & $8.3 \pm 4.5(1-15)$ & 0.048 \\
\hline
\end{tabular}

Values are presented as mean \pm standard deviation (range).

$\mathrm{UI}$, urinary incontinence; OABSS, overactive bladder symptom score; ICIQ-SF, International Consultation on Incontinence Questionnaire-Short Form.

\section{Clinical efficacy}

The subjective symptoms of UI improved for 13 patients (54.2\%). At base line, the frequency of UI was $13.2 \pm 12.4$ times (0.5-42 times) per week and at posttreatment the frequency of UI was 4.6 
\pm 8.2 times (0-21 times) per week, indicating a significant reduction $(\mathrm{P}=0.001)$ in the frequency of UI per week (Table 4$)$. An overactive bladder symptom score (OABSS) was obtained from 11 patients. The treatment did not provide a reduction in OABSS, which was $7.5 \pm 3.6(1-13)$ at baseline and $6.6 \pm 4.1(0-12)$ at posttreatment (Table 4 ). The ICIQ-SF at baseline was $10.5 \pm 5.1$ (1-18) and at posttreatment it was $8.3 \pm 4.4(1-15)$, indicating a reduction in the score for $\mathrm{UI}(\mathrm{P}=0.048)$ (Table 4$)$.

At baseline, the frequency of FI was $6.0 \pm 8.2$ times $(0.25-30$ times) per week and at posttreatment the frequency of FI was 1.6 \pm 2.1 times (0-7 times) per week, indicating a significant reduction ( $\mathrm{P}=0.005)$ (Fig. 2). Moreover, a reduction of $\geq 50 \%$ of FI per week was found in 14 of the patients (responders, 58.3\%). Ten patients did not reach a reduction of $\geq 50 \%$ (nonresponders, $41.7 \%$ ).

A FISI score was obtained from 23 patients and the number and percentage of the patients in each posttreatment status were as follows: a reduction in 14 patients (58.3\%), no change in 1 patient (4\%), and an increase in 7 patients (29.2\%). The Wexner score of 24 patients revealed that there was a reduction in 19 patients (76\%), no change in 3 patients (12.5\%), and an increase in 2 patients $(8.3 \%)$. The treatment provided a significant $(\mathrm{P}=0.003)$ reduction in FISI, which was $24.1 \pm 10.2(8-49)$ at baseline and 1.6 $\pm 2.1(0-7)$ at posttreatment (Fig. 3). The Wexner score at baseline was $11.6 \pm 3.5(3-17)$ and at posttreatment was $7.4 \pm 4.3(0-13)$, indicating a significant $(\mathrm{P}<0.0001)$ reduction in the score for $\mathrm{FI}$ (Fig. 4).

Twelve patients (50\%) in total, including 8 responders $(66.7 \%)$ and 4 nonresponders (33.3\%) completed the JFIQL questionnaire. The mean score for life style was $2.7 \pm 0.6(1.3-3.7)$ at base line and $2.9 \pm 0.9(1-4)$ posttreatment $(\mathrm{P}=0.092)$, coping/behavior was $2.5 \pm 0.7(1.4-4)$ at baseline and $2.7 \pm 0.8(1.1-4)$ at posttreatment $(\mathrm{P}=0.011)$, depression/self-perception was $2.8 \pm 0.8(1.3-$ $3.9)$ at baseline and $3.1 \pm 0.9(1.7-4.5)$ at posttreatment $(\mathrm{P}=$ $0.015)$ embarrassment was $2.1 \pm 0.7(1-3.5)$ at baseline and $2.6 \pm$ $0.8(1.3-4)$ at posttreatment $(\mathrm{P}=0.039)$, and the generic score was

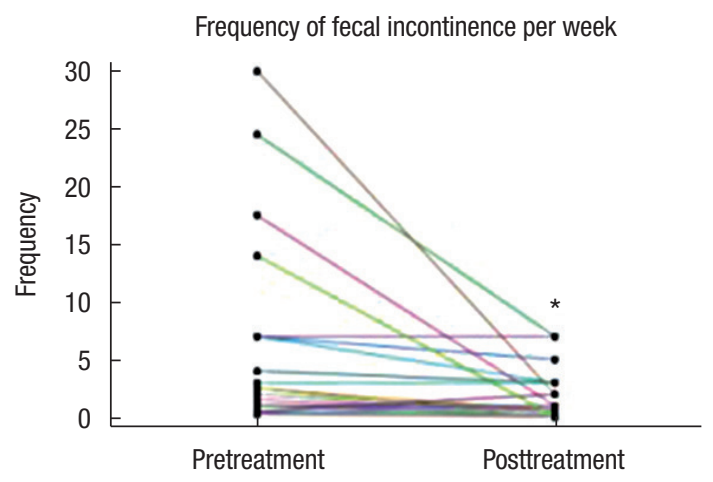

Fig. 2. Frequency of fecal incontinence per week $(\mathrm{n}=24)$. At baseline, the frequency of fecal incontinence (FI) per week was $6.0 \pm 8.2$ and at posttreatment the frequency of FI per week was $1.6 \pm 2.1$, indicating a significant reduction $\left({ }^{\star} \mathrm{P}=0.005\right.$ vs. the pretreatment period).
$2.5 \pm 0.1(1.5-3.4)$ at baseline and $2.8 \pm 0.7(1.5-3.8)$ at posttreatment $(\mathrm{P}=0.004)$. All of the items, except for lifestyle, significantly improved after treatment (Tables 1, 2).

The relationship between the responders and the background factors of FI was examined and a correlation was found between the responders; 14 of the patients over 75 years of age $(58.3 \%, \mathrm{P}=$ $0.005)$ and patients with comorbidity $(\mathrm{P}=0.005)$. The response rate was $80 \%$ among the elderly and $22 \%$ in patients 75 years of age or under $(n=9)$. There was no correlation between the responders and frequency of defecation, Bristol stool score, MRP, MSP, sphincter muscle dysfunction, improvement of UI symptom, and the type of FI (solid or liquid). A summary of the results is listed in Table 5.

Adverse events were seen in 4 patients (16.7\%) and they included stomach ache (1 patient), sensation of residual urine (1 patient), eyesight abnormality (1 patient), and dizziness (1 patient).

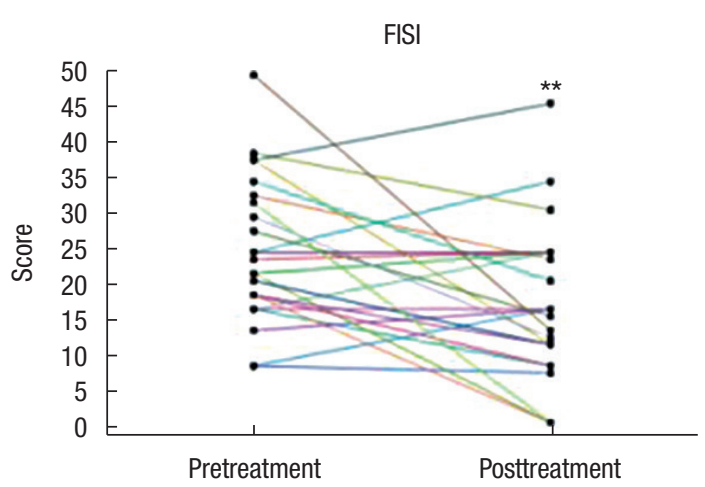

Fig. 3. Fecal Incontinence Severity Index (FISI) score $(\mathrm{n}=23)$. Propiverine treatment provided a significant $\left({ }^{* *} \mathrm{P}=0.003 \mathrm{vs}\right.$. the pretreatment period) reduction in scores between the pretreatment state $(24.1$ $\pm 10.2)$ and the posttreatment state $(16.3 \pm 10.9)$.

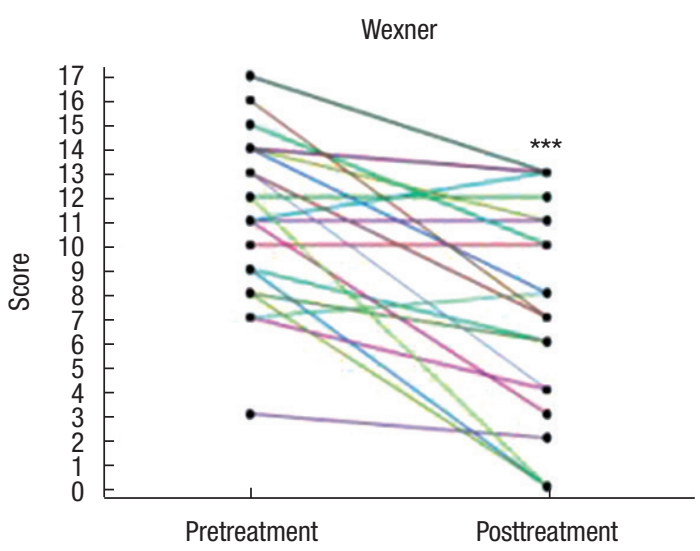

Fig. 4. Wexner score $(\mathrm{n}=24)$. Propiverine treatment provided a significant $\left({ }^{* * *} \mathrm{P}<0.0001\right.$ vs. the pretreatment period) reduction in scores between the pretreatment state $(11.6 \pm 3.5)$ and the posttreatment state $(7.4 \pm 4.3)$. 
Table 5. Comparison of the relationship between clinical findings and responder/nonresponder patients

\begin{tabular}{|c|c|c|c|c|}
\hline Variable & $\begin{array}{l}\text { All patients } \\
\text { (n) }\end{array}$ & $\begin{array}{l}\text { Responder } \\
\text { (n) }\end{array}$ & $\begin{array}{c}\text { Nonresponder } \\
\text { (n) }\end{array}$ & P-value \\
\hline Sex & & & & 0.067 \\
\hline Male & 7 & 6 & 1 & \\
\hline Female & 17 & 8 & 9 & \\
\hline Age (yr) & & & & 0.005 \\
\hline$\geq 76$ & 15 & 12 & 3 & \\
\hline$<76$ & 9 & 2 & 7 & \\
\hline Type of FI & & & & 0.172 \\
\hline Solid & 23 & 14 & 9 & \\
\hline Liquid & 1 & 0 & 1 & \\
\hline $\begin{array}{l}\text { Improvement of urinary } \\
\text { incontinence symptom }\end{array}$ & & & & 0.729 \\
\hline+ & 13 & 8 & 5 & \\
\hline- & 11 & 6 & 5 & \\
\hline $\begin{array}{l}\text { Treatment for Fl before this } \\
\text { study }\end{array}$ & & & & 0.233 \\
\hline+ & 16 & 8 & 8 & \\
\hline- & 8 & 6 & 2 & \\
\hline Anorectal, pelvic operation & & & & 0.484 \\
\hline+ & 10 & 5 & 5 & \\
\hline- & 14 & 9 & 5 & \\
\hline Comorbidity & & & & 0.005 \\
\hline+ & 10 & 9 & 1 & \\
\hline- & 14 & 5 & 9 & \\
\hline Bristol stool scale & & & & 0.126 \\
\hline$\geq 5$ & 9 & 7 & 2 & \\
\hline$<5$ & 15 & 7 & 8 & \\
\hline Anal sphincter defect & & & & 0.752 \\
\hline+ & 3 & 2 & 1 & \\
\hline- & 21 & 12 & 9 & \\
\hline $\mathrm{MRP}\left(\mathrm{cmH}_{2} \mathrm{O}\right)$, mean $\pm \mathrm{SD}$ & 18 & $46 \pm 18$ & $54 \pm 21$ & 0.594 \\
\hline $\mathrm{MSP}\left(\mathrm{cmH}_{2} \mathrm{O}\right)$, mean $\pm \mathrm{SD}$ & 17 & $176 \pm 107$ & $115 \pm 80$ & 0.242 \\
\hline
\end{tabular}

MRP, maximal anal resting pressure; MSP, maximal anal squeeze pressure; SD, standard deviation.

\section{DISCUSSION}

Previous studies have been conducted on the efficacy of PH for FI in Japan. However, this is the first study to evaluate the efficacy of $\mathrm{PH}$ as a possible treatment for FI. The findings revealed that $\mathrm{PH}$ reduced the frequency of FI in patients with both FI and UI. This study demonstrated the effectiveness of PH by 2 methods. First, $\mathrm{PH}$ caused a significant reduction in the frequency of FI per week.
Second, the scores produced by instrumentation (Wexner scale and FISI) to assess the severity of FI were significantly improved after treatment. Thus, PH may be effective for FI but the mechanism is still unclear. However, an assumption can be made that the pharmacological action for the anorectum and for the bladder are similar to each other.

$\mathrm{PH}$ is commonly used for the treatment of an overactive bladder, as it has direct influence on the smooth muscles of the urinary bladder by inhibiting calcium influx-induced muscle contraction [3] and its metabolites have an anticholinergic effect by inhibiting muscarinic receptors [4] of the bladder mucosa. Yoshida et al. [8] demonstrated the action mechanism of antimuscarinic drugs for an overactive bladder during the storage phase. The bladder urothelium releases many substances, including adenosine triphosphate (ATP) and acetylcholine (Ach) due to bladder distension during the storage phase and during stimulation. These substances stimulate afferent nerves and increase myogenic activity of the detrusor. The authors suggest that Ach from the urothelium may stimulate the muscarinic receptors on mucosa and cause the release of ATP in urothelium [8]. Various antimuscarinic drugs have inhibitory effects on ATP release and are therefore used for the treatment of overactive bladder. It has also been reported that the oxybutynin, propiverine, tolterodine, and solifenacin antimuscarinic drugs caused concentration dependent inhibition in ATP release, and the rank order of the maximum inhibition rate was propiverine $\geq$ solifenacin $\geq$ tolterodine $\geq$ oxybutynin [9]. Recently, PH has also been shown to be effective for patients with mixed urge and stress incontinence in daily practice. $\mathrm{PH}$ increases urethral pressure by inhibiting noradrenaline reuptake at the urethral level, and it may stimulate the smooth muscles of the bladder neck and proximal urethra through the alpha1 $\left(\alpha_{1}\right)$ adrenergic receptors [10]. Moreover, $\mathrm{PH}$ also stimulates the striated muscles of the urethra and pelvic floor by activating spinal motor neurons [10].

Ono et al. [11] reported that atropine can be used as an anticholinergic agent and that the muscarinic receptor antagonist significantly decreases the frequency of spontaneous contractions of the longitudinal muscle in the rat distal colon. $\mathrm{PH}$, used as an antimuscarinic receptor, may have a similar effect on the distal colon. The pharmacology of the internal anal sphincter was reviewed by Cook et al. [12]. They looked at the mechanism of contraction and relaxation of the internal anal sphincter muscle. The mechanism is basically a sympathetic input from the hypogastric plexus that releases noradrenaline and parasympathetic supply from the first, second, and third sacral nerves via the pelvic plexus and releases acetylcholine [12]. They suggest that the internal anal sphincter muscle contracts via the a1-adrenergic receptor and relaxes via the beta $\left(\beta_{2}\right)$-adrenergic receptor via nitric oxide. The muscarinic receptor is expressed on the nitric oxide-releasing nerve of the internal anal sphincter muscle [12]. The effect of PH as an antimuscarinic receptor and a1-adrenergic receptor is expected to have an antirelaxation effect on the internal anal sphinc- 
ter. A recent hypothesis has been proposed that suggests $\mathrm{PH}$ may be effective for FI as a pharmacological treatment because it slows colonic transit and reduces anal sphincter relaxation.

This study demonstrated that the patient responders tended to have a past history and comorbidities that included diabetes mellitus (3 patients), dementia (2 patients), lumber spinal canal stenosis ( 2 patients), history of lumber compression fracture (1 patient), and a history of spinal caries (1 patient). The surgical history of the responders included hemorrhoid surgery (1 patient) and total hysterectomy (1 patient). It is suspected that $\mathrm{PH}$ is more effective for patients who have a functional disorder rather than for those patients who have a structural defecation disorder, and it may have more of an effect on patients with FI in combination with UI. There were more elderly patients ( 75 years of age or older) among the responders than in the nonresponders, with no relation to comorbidity. However, some studies suggest that there may be a correlation between $\mathrm{PH}$ and age. Yoshida et al. [8] reported on the correlation between age and Ach release from human urothelium and the release was increased age-dependently. This is similar to our findings in this study that indicate bladder activity varies with aging. Nishijima et al. [13] found that PH had an effect on urinary urge incontinence in old rats but not in young rats. Moreover, $\mathrm{PH}$ did not increase serum catecholamine levels and changed the blood pressure and heart rate only in old rats. In this study, there were no significant adverse events, suggesting that $\mathrm{PH}$ may be suitable for use as treatment in the elderly.

The limitations of this study include not clarifying the classification of UI to stress UI or urge UI and the relatively small sample size. Because only 12 patients (50\%) completed the JFIQL questionnaire, it is difficult to conclude that $\mathrm{PH}$ improved patient QoL. PH is effective for FI but the mechanism is still unclear. Further randomized studies and clarification of the mechanism are needed before $\mathrm{PH}$ can be administered in clinical practice.

In conclusion, this study introduces a possible therapeutic option for the treatment of FI. PH reduced the frequency of FI in patients with both FI and UI. Responders tended to have a comorbidity and more elderly patients were among the responders than the nonresponders.

\section{CONFLICT OF INTEREST}

No potential conflicts of interest relevant to this article are reported.

\section{REFERENCES}

1. Norton C, Whitehead WE, Bliss DZ, Harari D, Lang J; Conserva- tive Management of Fecal Incontinence in Adults Committee of the International Consultation on Incontinence. Management of fecal incontinence in adults. Neurourol Urodyn 2010;29:199-206.

2. Paquette IM, Varma MG, Kaiser AM, Steele SR, Rafferty JF. The American Society of Colon and Rectal Surgeons' Clinical Practice Guideline for the treatment of fecal incontinence. Dis Colon Rectum 2015;58:623-36.

3. Haruno A, Yamasaki Y, Miyoshi K, Miyake H, Tsuchiya K, Kosa$\mathrm{ka} \mathrm{M}$, et al. Effects of propiverine hydrochloride and its metabolites on isolated guinea pig urinary bladder. Nihon Yakurigaku Zasshi 1989;94:145-50.

4. Nomura N, Kaneko S, Hamakawa T, Nagai M, Iriki M. Effects of propiverine hydrochloride (P-4) and its metabolites on urinary bladder function in anesthetized rats. Nihon Yakurigaku Zasshi 1989;94:173-80.

5. Rockwood TH, Church JM, Fleshman JW, Kane RL, Mavrantonis C, Thorson AG, et al. Patient and surgeon ranking of the severity of symptoms associated with fecal incontinence: the fecal incontinence severity index. Dis Colon Rectum 1999;42:1525-32.

6. Jorge JM, Wexner SD. Etiology and management of fecal incontinence. Dis Colon Rectum 1993;36:77-97.

7. Ogata H, Mimura T, Hanazaki K. Validation study of the Japanese version of the Faecal Incontinence Quality of Life Scale. Colorectal Dis 2012;14:194-9.

8. Yoshida M, Masunaga K, Nagata T, Yono M, Homma Y. The forefront for novel therapeutic agents based on the pathophysiology of lower urinary tract dysfunction: pathophysiology and pharmacotherapy of overactive bladder. J Pharmacol Sci 2010;112:12834.

9. Yoshida M, Masunaga K, Nagata T, Maeda Y, Yutaka Miyamoto, Junzo Kudoh, Yukio Homma. Attenuation of non-neuronal adenosin triphosphate release from human bladder mucosa by antimuscarinic agents. Low Urin Tract Dysfunct 2009;1:88-92.

10. Nishijima S, Sugaya K, Kadekawa K, Ashitomi K, Ueda T, Yamamoto H. Propiverine increases urethral wall catecholamine levels and bladder leak point pressure in rats. Int J Urol 2016;23:93-9.

11. Ono S, Mitsui R, Karaki S, Kuwahara A. Muscarinic and 5-HT4 receptors participate in the regulation of the frequency of spontaneous contractions of the longitudinal muscle in rat distal colon. Biomed Res 2005;26:173-7.

12. Cook TA, Brading AF, Mortensen NJ. The pharmacology of the internal anal sphincter and new treatments of ano-rectal disorders. Aliment Pharmacol Ther 2001;15:887-98.

13. Nishijima S, Sugaya K, Kadekawa K, Ashitomi K, Yamamoto H. Efficacy of propiverine, an anticholinergic agent, in young and old rats. Life Sci 2011;89:456-9. 